\title{
Systematic Design of Market-based Balancing Arrangements for Deregulated Power Systems: an Asynchronous Solution
}

\author{
J. H. Verberk, R. M. Hermans, P. P. J. Van den Bosch, A. Jokić, J. Frunt
}

\begin{abstract}
In the deregulated electrical energy market, network operators have to provide market participants with appropriate incentives to guarantee stable operation of the power grid. We demonstrate that the current synchronous energy-based market and incentive system do not necessarily induce power exchange profiles that contribute to grid stability and security of supply. State-of-the-art solutions for tackling the inconsistency between energy-based market mechanisms and power-related balancing objectives can decrease freedom of trade or increase market complexity. This paper provides an alternative scheduling concept as a means to overcome this issue, which relies on asynchronous settlement of energy transactions. We show that in this way, grid operation can become more robust and the strain on balancing reserves can be reduced considerably.
\end{abstract}

Index Terms-Deregulated electrical energy markets, power generation dispatch, power system control

\section{INTRODUCTION}

A $\mathrm{N}$ important prerequisite for reliable operation of the electricity grid is that power supply and demand are balanced all the time, as efficient methods for storing large quantities of electrical energy are scarce. Before liberalization of the electricity market, the electrical energy and power balance was controlled in a rather straightforward way, as a small group of operators had direct control over generation. A large part of the energy production could efficiently be scheduled to minimize the expected imbalance with respect to the rather well-predictable load, whereas simple classical feedback control techniques sufficed for compensating the relatively small amount of unforeseen imbalance fluctuations during the operational day.

Today, this situation has changed drastically. In the deregulated European electricity system, generators are owned and controlled by multiple market actors that compete for supply and demand of energy under the supervision of a publiclyregulated Transmission System Operator (TSO). The TSO is responsible for the electrical transmission infrastructure and thus for preserving the real-time power balance. It should achieve this by providing Balance Responsible Parties (BRPs) with such incentives that rational, i.e., profit-driven, behavior on their side leads to reliable operation of the network, see, e.g., [1].

Since the main electricity market commodity is energy rather than power (which is a flow variable of undefined economical value), the TSO can only provide energy-based financial incentives, even though control objectives actually

J. H. Verberk, R. M. Hermans, P. P. J. Van den Bosch, A. Jokić and J. Frunt are with the department of Electrical Engineering, Eindhoven University of Technology, P.O. Box 513, 5600 MB Eindhoven, The Netherlands, E-mails: \{r.m.hermans, p.p.j.v.d.bosch, a.jokic, j.frunt\}@tue.nl. require balancing of power. Moreover, because energy transactions are defined with respect to fixed-length time slots or Program Time Units (PTUs), the market and TSO can only settle energy imbalances at a limited number of discrete time instants. Recent studies show that already today, the inconsistency between periodic energy-based settlement on one hand and power-related stability requirements on the other is causing severe frequency deviations in the European power network, particularly at PTU boundaries, see [2]-[4].

The contribution of this paper is twofold. Firstly, we analyze the fundamental flaws of the current European market arrangements with respect to grid stability and balancing goals, in particular, by recalling the strong dependency between market-induced generation set-point interchanges and power imbalance fluctuations at PTU boundaries. Secondly, a method is proposed to loosen the coupling between the incentive/market layer and the physical network layer as a solution to these issues. This method relies on an asynchronous energy transaction settlement system that yields a short, virtual PTU length on the overall, aggregated network level, whereas the PTU length observed by individual market actors is not affected. This enables the TSO to decrease the expected openloop power imbalance, as energy supply and demand expectations acquired during the scheduling/dispatch phase can be used more efficiently during network operation, with minor consequences for trade complexity and market freedom. Openand closed-loop simulation results are provided to compare the performance of the proposed asynchronous settlement concept with that of state-of-the-art approaches. We finish with conclusions.

Nomenclature: Power [MW] and energy [MWh] can both be positive or negative; they are either delivered $(>0)$ or withdrawn $(<0)$ from the grid. Let $\mathbb{N}, \mathbb{N}_{+}, \mathbb{R}$ and $\mathbb{R}_{+}$denote the field of integers $\{\ldots,-1,0,1, \ldots\}$, the set of strictly positive integers $\{1,2, \ldots\}$ and the set of real and nonnegative real numbers, respectively. For every $c \in \mathbb{R}$, let $|c|$ denote its absolute value and let $|c|=\max \{k \in \mathbb{N} \mid k \leq c\}$. The first and second order derivatives of some continuous function $f: \mathbb{R} \rightarrow \mathbb{R}$ are denoted by $f^{\prime}$ and $f^{\prime \prime}$, respectively.

\section{INCONSISTENCY OF}

\section{Market ArRangements and Balancing ObJectives}

So far, the present market and transaction-settlement arrangements have proven to be reasonably adequate for ensuring balanced and stable operation of the European power system, see, for example, [5]. However, it is possible to show that the present way of dealing with uncertainty during forward trading is neither consistent, nor well-suited for the 
challenges that power networks face in the future, such as the increased extent of fluctuations in power supply due to renewable sources.

One of the objectives of forward trading/scheduling is to guarantee that for each PTU, production and consumption are expected to be balanced. Since energy, rather than power, is traded on the electricity market, the market condition for openloop balance is formulated in terms of energy, i.e.,

$$
\sum_{i=0, \ldots, N-1} E_{i}[n]=0, \quad n \in \mathbb{N},
$$

where $E_{i}[n][\mathrm{MWh}]$ denotes the nett energy transaction of balance responsible party $i \in \mathcal{I}_{\mathrm{BRP}}:=\{0,1, \ldots, N-1\}$ during the $n$-th PTU.

Let the controllable and uncontrollable power prosumption (production/consumption) of BRP $i$ at time $t \in \mathbb{R}$ be denoted by $P_{i}(t)[\mathrm{MW}]$ and $\mu_{P, i}(t)[\mathrm{MW}]$, respectively. To comply with the ahead-established market transactions (and thus, to avoid imbalance costs), BRPs can track any power profile $P_{i}(t)$ that satisfies $E_{i}[n]=\int_{\text {PTU } n}\left\{P_{i}(t)+\mu_{P, i}(t)\right\} d t$. However, for BRPs with strictly convex power prosumption costs, the most profitable way to supply a certain amount of energy is to keep controllable power prosumption constant; see, e.g., [6]. These BRPs therefore attempt to generate the step-wise averaged power profiles

$$
\widetilde{P}_{i}(t)=\widetilde{P}_{i}[n], \quad \text { for } t \in\left[t_{n}, t_{n+1}\right)
$$

where

$$
\widetilde{P}_{i}[n]:=\frac{1}{T_{\mathrm{PTU}}}\left(E_{i}[n]-\int_{t_{n}}^{t_{n+1}} \mu_{P, i}(t) d t\right),
$$

and where $T_{\mathrm{PTU}}[\mathrm{s}]$ denotes the PTU-length and $t_{n}:=n T_{\mathrm{PTU}}$, $n \in \mathbb{N}$. Now suppose that BRPs are able to track $\widetilde{P}_{i}(t)$ exactly, and let $\mu_{P, i}(t)$ be piecewise constant with changes at $t_{n}$. This is depicted on the left-hand side of Fig. 1, which shows the total power generation $P(t):=\sum_{i} P_{i}(t)=\widetilde{P}(t):=\sum_{i} \widetilde{P}_{i}(t)$, the total noncontrollable prosumption $\mu_{P}(t):=\sum_{i} \mu_{P, i}(t)$ and their sum, i.e., the open-loop aggregated power imbalance

$$
\Delta P_{\mathrm{OL}}(t):=P(t)+\mu_{P}(t) .
$$

In this ideal case, open-loop energy balance condition (1) coincides with an open-loop power balance $\left(\Delta P_{\mathrm{OL}}=0\right.$ for all $t \in \mathbb{R}$ ). However, in reality, power demand profiles will never be step-wise; they are smooth due to generator inertia and other physical restrictions. This is schematically illustrated in the right-hand side of Fig. 1, which shows that in practice, $\Delta P_{\mathrm{OL}}(t)$ is nonzero and especially large at setpoint interchange instants $t_{n}$, i.e, at PTU boundaries. Thus, although the present market and incentive system complies with requirement (1) for open-loop energy balance (at discrete time instants $t_{n}$ ), it introduces large open-loop mismatches in terms of power, which need to be compensated by considerable control effort in real-time, even if no external disturbances act on the system.

Note that the effects caused by the above described inconsistency are already observable today. Fig. 2 shows a frequency measurement performed in the European/ENTSO-E synchronous grid during the evening, see [7]. Note that directly

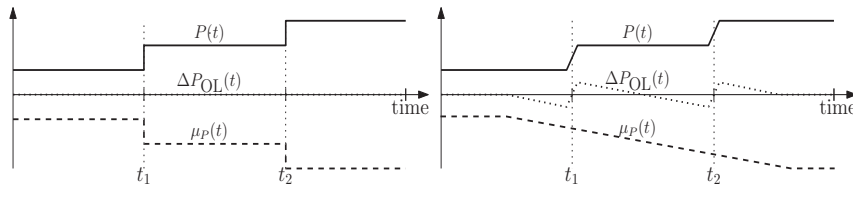

Fig. 1. Open-loop power imbalance for step-wise/smooth exchange profiles.

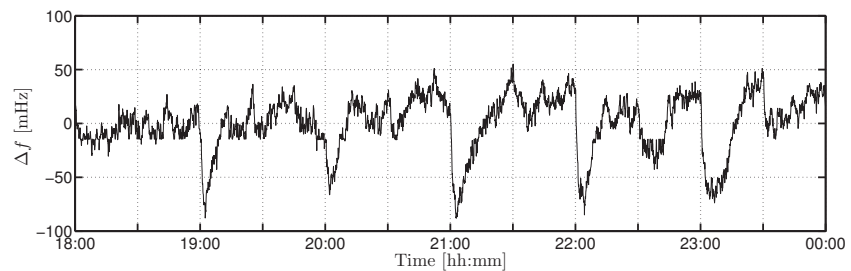

Fig. 2. Grid frequency measurement, reproduced from [7].

after a change in energy exchange set-points, which occurs every full hour, large frequency deviations occur. These deviations due to step-wise power reference tracking activate a significant share of primary and secondary control (PC/SC) reserves, such that the system becomes vulnerable to disturbances, see [2]-[4].

\section{A. State-of-the-art solutions}

Because the above explained power imbalance fluctuations are expected to increase in the near future, e.g., due to the growth of energy trading volumes and the large-scale integration of renewable sources, a number of solutions have been proposed in the literature, see, for example, [2], [3]. These approaches are briefly discussed below.

1) Explicit constraints on power: A natural way to decrease the open-loop power imbalance is to put an upper bound on the ramp rates of $P_{i}(t)$, see [2]. This constraint (on power instead of energy) prevents large step-wise changes in $P(t)$ at PTU boundaries, such that intra-PTU power imbalances can be decreased effectively. However, due to the integral relation of power and energy, this simultaneously limits market activity, and the implementation of power-based incentives and verification of the associated conditions is difficult.

2) Shorter PTUs: Shorter PTUs generally lead to smaller average differences in the amounts of energy exchanged during subsequent PTUs. In accordance with (2), this results in decreased step-wise changes at PTU boundaries, see [3]. However, as energy transactions are settled on a PTU basis, a decrease in PTU length will increase the number of transactions correspondingly. This will increase scheduling and trade complexity. Moreover, it is difficult for BRPs to respond to portfolio deviations (and, correspondingly, to avoid imbalance costs) within short PTUs during the operational day. As a consequence, the support for decreasing the PTU length may be low, particularly amongst BRPs.

\section{Asynchronous PRogram TIME Units}

To the best of our knowledge, decreasing the PTU length is the only state-of-the-art solution that can tackle the fundamental flaw of the current (forward) balancing arrangements, i.e., 
the inconsistency between energy-based trade and power-based balancing requirements. However, the increase of market complexity associated with straightforwardly shortening the PTU length renders this solution unsuited for implementation. In this section we show that it is still possible to increase the PTU lattice density, and hence, open-loop balancing performance, without affecting forward trade, by adopting an asynchronous settlement method.

Let $M \leq N$ partitions or time frames $\mathcal{P}_{j}, j=0, \ldots, M-1$, of the continuous-time axis $\mathbb{T}=\mathbb{R}$ be given such that

$$
\mathbb{T}=\left\{\ldots, \mathbb{T}_{j}[-1], \mathbb{T}_{j}[0], \mathbb{T}_{j}[1], \ldots\right\},
$$

with partition blocks (or time-shifted PTUs)

$$
\mathrm{T}_{j}[n]:=\left[\left(\varphi_{j}+n\right) T_{\mathrm{PTU}}, \quad\left(\varphi_{j}+n+1\right) T_{\mathrm{PTU}}\right),
$$

for $n \in \mathbb{N}$, where $\varphi_{j}:=\frac{(1+2 j)}{2 M}$. Each partition $\mathcal{P}_{j}$ is assigned to a nonempty set of BRPs $\mathcal{I}_{j} \subseteq \mathcal{I}_{\mathrm{BRP}}$, with $\mathcal{I}_{j}$ constructed in such a way that $\bigcup_{j=0, \ldots, M-1} \mathcal{I}_{j}=\mathcal{I}_{\mathrm{BRP}}$ and $\mathcal{I}_{j} \cap \mathcal{I}_{l}=\emptyset$ whenever $j \neq l, j, l \in\{0,1, \ldots, M-1\}$. Next, consider the following algorithm.

\section{Algorithm III.1 (Asynchronous PTU Method (APM))}

1) Trade: Each BRP $i \in \mathcal{I}_{B R P}$ submits its $T_{P T U}$-based Eprograms/transactions $E_{i}[n]$ for the upcoming day to the TSO, who performs a consistency check according to (1).

2) Scheduling: Given a set of consistent transactions $E_{i}[n]$, $i \in \mathcal{I}_{B R P}$, the TSO reschedules them to obtain E-references that are defined with respect to the time frames $\mathcal{P}_{j}$. That is, it expects BRP $i \in \mathcal{I}_{j}$ to exchange a nett amount of energy with the grid in time interval $\mathbb{T}_{j}[n]$ of

$$
\hat{E}_{i}[n]:=\left(1-\varphi_{j}\right) E_{i}[n]+\varphi_{j} E_{i}[n+1] .
$$

3) Settlement: Any deviations from $\hat{E}_{i}[n]$ within $\mathbb{T}_{j}[n]$ are settled in a fashion that is similar to the present arrangements, except for the different PTUs used per group of BRPs $\mathcal{I}_{j}$.

The novelty of Alg. III.1 completely lies in the scheduling and settlement step. Thus, even though APM employs asynchronous settlement of energy transactions, energy trade itself is still based on standard, synchronized PTU intervals $\left[t_{n}, t_{n+1}\right)$. Hence, BRPs may establish transactions with any other market actor, regardless of their respective time frames during the operational day. Also, note that the E-references $\hat{E}_{i}$ are dependent on the synchronous E-programs $E_{i}$ only, such that no prosumption is shifted from one BRP to an other.

Remark III.2 The time shifts employed by APM, i.e. $\varphi_{j} T_{\mathrm{PTU}}=\frac{(1+2 j)}{2 M} T_{\mathrm{PTU}}$, are nonzero for all $j=0, \ldots, M-1$. This avoids possible competitive advantages arising from market-synchronized settlement.

In what follows, it is assumed that production costs are strictly convex functions of power prosumption, and all BRPs respond to incentives in a rational, cost-minimizing fashion. These assumptions are widely used in power system economics, see [8]. Moreover, for simplicity, let $M=N$ and

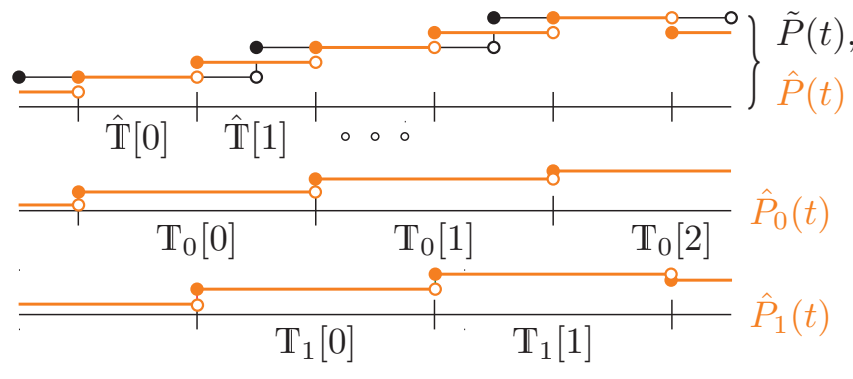

Fig. 3. Controllable power prosumption under APM, for $M=N=2$.

$\mathcal{I}_{j}:=\{j\}$, such that the energy transactions of BRP $i$ are settled with respect to time frame $\mathcal{P}_{i}$. Then, (6) reduces to

$$
\hat{E}_{i}[n]:=\left(1-\varphi_{i}\right) E_{i}[n]+\varphi_{i} E_{i}[n+1] .
$$

Now consider the following proposition.

Proposition III.3 Rescheduling of energy prosumption according to (6) does not affect the nett energy exchange for the network as a whole.

Prop. III.3 is derived as follows. Consider a sequence of PTUs $n=0 \ldots, K-1$, for which the total energy exchange of BRP $i$ under synchronous/asynchronous scheduling satisfies

$$
\begin{aligned}
& \sum_{n=0}^{K-1} E_{i}[n]=\sum_{n=0}^{K-1} \varphi_{i} E_{i}[n]+\left(1-\varphi_{i}\right) E_{i}[n], \\
& \sum_{n=0}^{K-1} \hat{E}_{i}[n]=\sum_{n=0}^{K-1}\left(1-\varphi_{i}\right) E_{i}[n]+\varphi_{i} E_{i}[n+1] .
\end{aligned}
$$

Thus, the nett difference in energy exchange is given by

$$
\Delta E_{i}(K):=\sum_{n=0}^{K-1} E_{i}[n]-\hat{E}_{i}[n]=\varphi_{i}\left(E_{i}[0]-E_{i}[K]\right) .
$$

In practice, the sequence of energy transactions will be highly periodic (e.g., over a day or a year). Hence, it follows that, for appropriate $K^{*}, E_{i}[0] \approx E_{i}\left[K^{*}\right]$ and thus $\Delta E_{i}\left(K^{*}\right) \approx 0$. Moreover, in accordance with (6), all transactions $E_{i}[n]$ are distributed over a period $\left[\left(\varphi_{i}+n-1\right) T_{\mathrm{PTU}},\left(\varphi_{i}+n+1\right) T_{\mathrm{PTU}}\right)$ of length $2 T_{\mathrm{PTU}}$. Note that this interval includes the PTU that was used for establishing $E_{i}[n]$, i.e., $\left[m T_{\mathrm{PTU}},(n+1) T_{\mathrm{PTU}}\right)$. Since no transactions are shifted from one BRP to another, equivalence of total BRP energy exchange yields equivalence of total aggregated energy exchange, and Prop. III.3 follows.

Next, we recall that under APM, the controllable generators of rational BRPs will track step-wise power profiles $\hat{P}_{i}(t)=$ $\hat{P}_{i}[n]$ for $t \in \mathbb{T}_{i}[n]$, with

$$
\hat{P}_{i}[n]:=\frac{1}{T_{\mathrm{PTU}}}\left(\hat{E}_{i}[n]-\int_{t \in \mathbb{T}_{i}[n]} \mu_{P, i}(t) d t\right),
$$

to comply with E-reference-based portfolios in a similar way as BRPs comply with E-programs under the present scheduling arrangements, due to strict convexity of the prosumption costs. The resulting controllable power exchange profile for the network as a whole, i.e., the aggregation of individual timeshifted step-wise power profiles, is given by

$$
\hat{P}(t)=\hat{P}[k]:=\sum_{i=0}^{N-1} \hat{P}_{i}\left[\left\lfloor\frac{k-i}{N}\right\rfloor\right] \quad \text { for } t \in \hat{\mathbb{T}}[k],
$$


where $\hat{\mathbb{T}}[k]:=\left[\frac{1+2 k}{2 N} T_{\mathrm{PTU}}, \frac{1+2(k+1)}{2 N} T_{\mathrm{PTU}}\right)$. Fig. 3 illustrates this aggregation for $N=2$. Note that even though the prosumption profiles of individual BRPs are piecewise constant with steps of length $T_{\mathrm{PTU}}$, the corresponding nett prosumption on the aggregated system level is less coarse with steps of length $\frac{T_{\mathrm{PTU}}}{N}$. Hence, in contrast to standard, synchronized scheduling, APM can induce an aggregated controlled power exchange profile that captures the smooth dynamics of $\mu_{P}(t)$ well and yields low open-loop imbalance, at any time instant $t \in \mathbb{R}$, in terms of both energy and power. The effectiveness of APM is dependent on the distribution of energy transactions over the individual BRPs, however. In what follows, we will describe two extreme scenarios to illustrate this dependency.

Firstly, suppose that BRP 0 is responsible for all controllable prosumption in the network. Accordingly, the aggregated controllable power prosumption $\hat{P}(t)=\hat{P}_{0}(t)=\hat{P}_{0}[n]$ for $t \in\left[\left(\varphi_{0}+n\right) T_{\mathrm{PTU}},\left(\varphi_{0}+n+1\right) T_{\mathrm{PTU}}\right)$ is step-wise with step width $T_{\mathrm{PTU}}$, both under the present arrangements and under APM. Thus, in this case, APM does not improve open-loop balancing performance.

Next, consider a second scenario where the controllable energy prosumption is uniformly distributed over the BRPs $i=0, \ldots, N-1$, and let BRP 0 be responsible for all noncontrollable prosumption (i.e., $\mu_{P, i}(t) \neq 0$ only for $i=0$ ). Hence, $T_{\mathrm{PTU}} \widetilde{P}_{i}[n]=-\frac{1}{N} \int_{t_{n}}^{t_{n+1}} \mu_{P, 0}(t) d t$ for all $i \in \mathcal{I}_{\mathrm{BRP}}$. Moreover, suppose that

$\int_{t \in \mathbb{T}_{0}[n]} \mu_{P}(t) d t=\left(1-\varphi_{0}\right) \int_{t_{n}}^{t_{n+1}} \mu_{P}(t) d t+\varphi_{0} \int_{t_{n+1}}^{t_{n+2}} \mu_{P}(t) d t$.

It follows from (7)-(9) that

$$
\hat{P}[k]=\frac{1}{N} \sum_{i=0}^{N-1}\left(1-\varphi_{i}\right) \widetilde{P}\left[\left\lfloor\frac{k-i}{N}\right\rfloor\right]+\varphi_{i} \widetilde{P}\left[\left\lfloor\frac{k-i}{N}\right\rfloor+1\right],
$$

which is a weighted sum of time-shifted economically optimal prosumption profiles for the present, synchronous settlement arrangements. From (10) it follows that in this second scenario, the aggregated controllable power production profile is stepwise with a step width of $\frac{T_{\mathrm{PTU}}}{N}$. Moreover, in Appendix A it is shown that (10) is equivalent to convolving (2) with a digital low-pass finite-impulse response filter. This filter is specified by the coefficient vector/impulse response

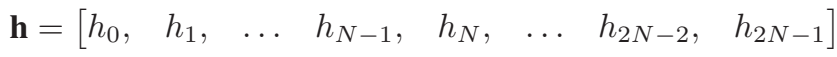

$$
\begin{aligned}
& =\frac{1}{2 N^{2}}[1,1+2, \ldots 1+2(N-1), 1+2(N-1), \ldots 1+2,1] \text {. }
\end{aligned}
$$

The low-pass character of APM ensures a smooth adjustment of controllable generation on the aggregated power system level. Instead of interchanging controllable prosumption setpoints simultaneously at market-PTU boundaries, BRPs will adjust their production at different time instances, thus effectively tackling the issues discussed in Sect. II.

Although, intuitively, the smoothing/de-synchronizing effect is expected to be optimal for evenly distributed controllable prosumption capacity, APM will improve the open-loop balancing performance as long as the controllable load/generation is rescheduled over more than one time frame $\mathcal{P}_{i}$. This observation is formalized as follows.
Proposition III.4 APM provides BRPs with an incentive for delivering power in such a way that the aggregated dispatch profile yields an open-loop power imbalance that is at most as large as, but (under normal operating conditions) most likely less than the imbalance attained by a synchronous method with identical PTU length.

The details on deriving Prop. III.4 are provided in Appendix B; below, we list the main results only.

The worst-case upper bound on the open-loop power mismatch attained by APM is

$$
\left|\Delta P_{\mathrm{OL}}(t)\right|_{N=1}\left|\leq \frac{T_{\mathrm{PTU}}}{2}\right| \mu_{P}^{\prime}(t)\left|+\frac{T_{\mathrm{PTU}}^{2}}{6}\right| \mu_{P}^{\prime \prime}(t) \mid+\mathcal{O}\left(T_{\mathrm{PTU}}^{3}\right),
$$

see Appendix B. As explained above, this bound applies to the current, synchronous settlement arrangements, or, equivalently, to APM with $N=1$ /with all controllable energy prosumption concentrated in one sequence. The best-case upper bound on the open-loop imbalance, as obtained for uniform distribution of controllable exchange and $N \rightarrow \infty$, is

$$
\left|\Delta P_{\mathrm{OL}}(t)\right|_{N \rightarrow \infty}\left|\leq \frac{T_{\mathrm{PTU}}^{2}}{6}\right| \mu_{P}^{\prime \prime}(t) \mid+\mathcal{O}\left(T_{\mathrm{PTU}}^{3}\right) .
$$

From (12) and (13) follows that APM gives an approximation of the actual noncontrollable power prosumption profile that is at least as accurate as the profile induced by synchronous arrangements, which supports Prop. III.4.

Remark III.5 Prop. III.4 shows that a balanced distribution of controllable generation/load over the time frames $\mathcal{P}_{j}$ is crucial for optimizing APM's performance. Thus, a natural way of assigning the BRPs to the different partitions is based on their share of the total controllable prosumption capacity.

Remark III.6 In contrast to what one might expect, the openloop power imbalance $\Delta P_{\mathrm{OL}}(t)$ attained by APM does not converge to zero for all $t \in \mathbb{R}$ if $N \rightarrow \infty$. This issue is inherent to establishing PTU-based energy (instead of continuous-time power) transactions on the market, which can be considered as sampling the smooth, expected noncontrollable prosumption profiles at a nonuniform sampling rate. This is explained below.

The mean value theorem states that for continuous, differentiable $\mu_{P}(t)$, there exists a $t_{n}^{*} \in\left[t_{n}, t_{n+1}\right)$ such that

$$
\int_{t_{n}}^{t_{n+1}} \mu_{P}(t) d t=\left(t_{n+1}-t_{n}\right) \mu_{P}\left(t_{n}^{*}\right)=T_{\mathrm{PTU}} \mu_{P}\left(t_{n}^{*}\right) .
$$

In other words, $\mu_{P}(t)$ takes on its average value $\widetilde{P}[n]$ at some point of the $n$-th PTU, and thus, all $\widetilde{P}_{i}[n]$ are (weighted) samples of $\mu_{P}(t)$ taken at this unknown time instant $t_{n}^{*} \in$ $\left[t_{n}, t_{n+1}\right)$. This corresponds to nonuniform sampling of $\mu_{P}(t)$ at an average rate of $\frac{1}{T_{\mathrm{PTU}}}$.

In the literature, many methods are available for reconstructing nonuniformly, super-Nyquist sampled signals, see, e.g., [9]. Crucial to these approaches is that the sample instants $t_{n}^{*}$ are distinct and known. PTU-based trade does not satisfy the latter condition. The nonuniform samples $E_{i}[n]$ are therefore not sufficient for perfect reconstruction of the power profile $\mu_{P}(t)$, and accordingly, the bound on the corresponding open-loop power imbalance $\Delta P_{\mathrm{OL}}(t)$ does not converge to 0 as $N \rightarrow \infty$. 


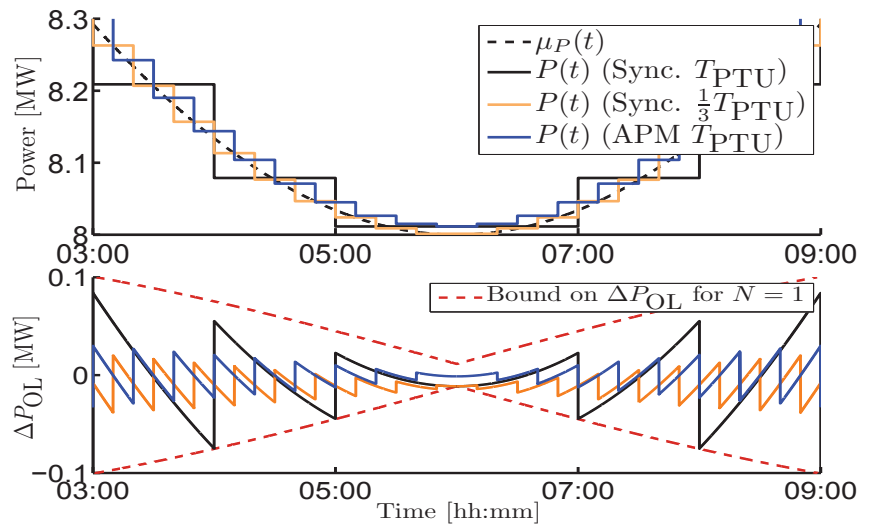

Fig. 4. Open-loop power exchange and imbalance profiles for $N=3$.

\section{Simulation Results}

The effectiveness of APM is evaluated by comparing it with synchronous scheduling for different PTU lengths. We begin with open-loop scheduling performance, followed by a comparison of closed-loop simulation results.

\section{A. Open-loop performance}

The open-loop performance of a $T_{\mathrm{PTU}}$ and a $\frac{T_{\mathrm{PTU}}}{N}$ PTUlength synchronous settlement scheme are compared with the performance attained by an evenly-distributed $N$-sequence APM scheme with a PTU-length of $T_{\text {PTU }}$. The asynchronous scheme is simulated for $N=3$ and $N=1000$, where the latter scenario provides an indication of the results for $N \rightarrow \infty$. In the simulation, for simplicity, the noncontrollable power exchange profile $\mu_{P}(t)$ is assumed to be sinusoidal with a period of one day.

Fig. 4 shows the open-loop performance of the current arrangements, that of a synchronous scheme with PTU length $\frac{T_{\text {PTU }}}{3}$ and that of a 3-sequence asynchronous scheme. The upper bound on the open-loop error $\left|\Delta P_{\mathrm{OL}}(t)\right|$ for the synchronous scheme, i.e., (12), is represented by the dashed lines in the lower subfigure. Both the APM and the synchronous scheme with PTU length $\frac{T_{\mathrm{PTU}}}{3}$ decrease the worst-case open-loop error by a factor of approximately 3. Furthermore, note that the open-loop error resulting from $\frac{T_{\mathrm{PTU}}}{3}-\mathrm{PTU}$ scheduling is 0 whenever $\frac{\mathrm{d} \mu_{P}}{\mathrm{~d} t}(t)=0$ (for instance, at $06: 00 \mathrm{~h}$ ), whereas a small error is apparent in the asynchronous case, due to nonzero higher order derivatives in (13).

Fig. 5 shows the simulation results for $N=1000$. The worst-case open-loop error envelope for the $N \rightarrow \infty$ APM settlement scheme, i.e., (13), is shown in the bottom plot. Although increasing $N$ does not reduce the open-loop APM error to zero (see Remark III.6), it is possible to get arbitrarily close to error bound (13) for all $t \in \mathbb{R}$ by choosing appropriate $N \in \mathbb{N}_{+}$.

Now consider the root mean square imbalance/error criterion $e(T):=\sqrt{\int_{0}^{T}\left|\Delta P_{\mathrm{OL}}(t)\right|^{2} d t}$. Evaluating this criterion for a period of one day ( $T=86400 \mathrm{~s}$ ) and $N=3$ yields a $64 \%$ and $67 \%$ reduction in $e(T)$ for APM and $\frac{T_{\text {PTU }}}{3}$-based synchronized settlement, respectively, measured with respect to the error induced by the standard $T_{\mathrm{PTU}}$-based synchronous

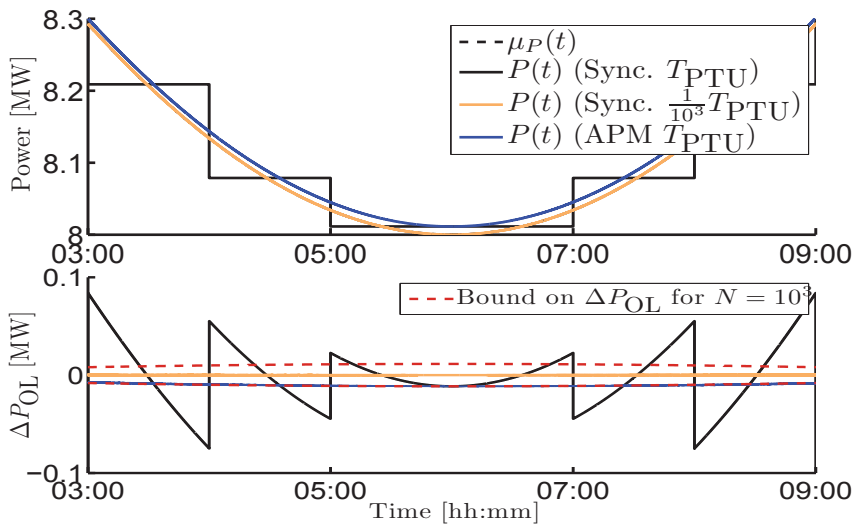

Fig. 5. Open-loop power exchange and imbalance profiles for $N=1000$.

scheme. For $N=1000$ this reduction is even larger, that is, $85 \%$ and $99 \%$, respectively. These figures show that, even for relatively small values of $N$, both the APM-based and short-PTU schemes can outperform the current arrangements. However, although APM and PTU-shortening lead to similar improvement of the open-loop balancing quality, the complexity of their implementation is quite different. In the shortened PTU case, large $N$ values correspond to small PTU-lengths, and thus require highly accurate prediction models and many decisions for trade. This is not the case for the APM scheme, as the corresponding market is based on a PTU-length of $T_{\mathrm{PTU}}$ for any $N \in \mathbb{N}_{+}$.

\section{B. Closed-loop performance}

Next, we focus on real-time balancing. Fig. 6 schematically depicts the simulated 5-BRP benchmark power network. Its closed-loop performance is evaluated during regular, unperturbed operation under the current, synchronized (ENTSO-E) settling arrangements and for APM with $N=5$ and evenlydistributed energy transactions. All BRPs are described by a linear, lumped generator model, reproduced from [10] and schematically shown in Fig. 7. Frequency/imbalance control is implemented by two parallel feedback loops. The primary control law is $P_{\mathrm{PC}}(t)=-R D(\Delta f(t))$, with frequency deviation $\Delta f(t):=f(t)-f_{0}, f_{0}:=50 \mathrm{~Hz}$, and with dead-band

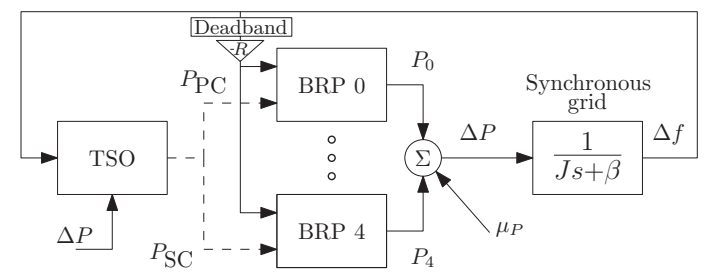

Fig. 6. Benchmark power system.

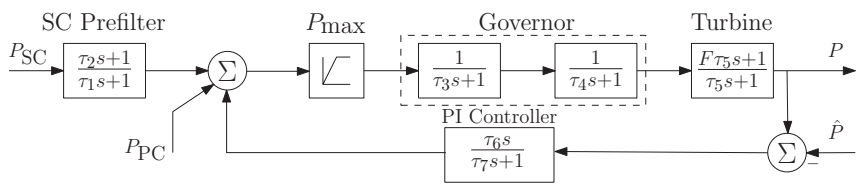

Fig. 7. Linear BRP/generator model. 


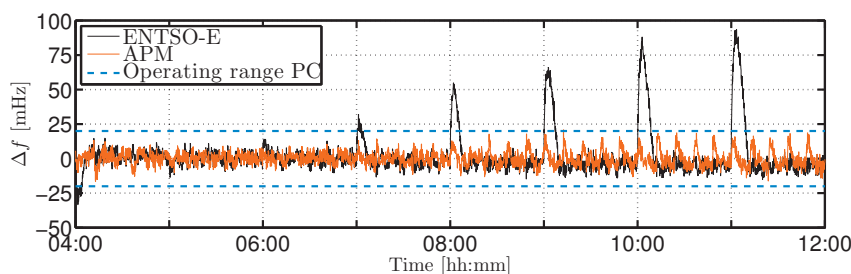

Fig. 8. Evolution of the closed-loop grid frequency over time.

function

$$
D(x):= \begin{cases}0 & \text { for }|x| \leq d \\ x & \text { for }|x|>d\end{cases}
$$

where $d \in \mathbb{R}_{+}$is some operating threshold. Secondary control power $P_{\mathrm{SC}}(t)$ is requested by the TSO through the classical feedback law $P_{\mathrm{SC}}(t)=K_{\mathrm{p}, \mathrm{SC}} \mathrm{ACE}(t)+K_{\mathrm{i}, \mathrm{SC}} \int \mathrm{ACE}(t) d t$, where $\operatorname{ACE}(t):=\Delta P(t)+K_{f} \Delta f(t)$ is the area control error. All network parameters, such as the total inertia $J$, the loaddamping coefficient $\beta$, the primary control operating range $d$ and the gains of the primary and secondary control loops are taken from [11]. BRP models 0-3 are dimensioned as (relatively slow) coal-fired generators; BRP 4 is modeled as a fast-responding gas-fueled power plant. All BRPs have equal production capacities $P_{\max }$.

The closed-loop performance is measured in terms of the maximum frequency deviation $\Delta f_{\max }:=\max _{t}\left|f(t)-f_{0}\right|$ and the primary and secondary control effort, defined as $E_{x}(T):=\int_{0}^{T}\left|P_{x}(t)\right| d t[\mathrm{GWh}$, where $x$ can be either "PC" or "SC" to denote primary or secondary balancing power/energy, respectively. Table I lists the corresponding performance results; Fig. 8 depicts the evolution of the grid frequency over time. Firstly, note that the synchronous settlement result in Fig. 8 shows a clear resemblance with the actual ENTSOE-grid measurements shown in Fig. 2, thus indicating that the simulation captures the major power system dynamics well. Secondly, it can be observed that the grid frequency is stabilized, i.e., is driven back to $f_{0}$ in case of disturbances, which, in this scenario, completely originate from the mismatch between the smooth sinusoidal load profile and the aggregated step-wise generation schedules. Table I shows that the daily APM-induced maximum frequency deviations are smaller than the deviations caused by the synchronous scheme. Moreover, as the closed-loop power imbalance induced by asynchronous settlement completely lies within the dead-band interval $[-d, d]$, APM avoids undesired activation of fast, primary balancing reserves. Also, the simulation illustrates that asynchronous scheduling reduces the need for secondary control energy, due to the corresponding improvement in openloop scheduling efficiency.

TABLE I

ASSESSMENT OF CLOSED-LOOP PERFORMANCE

\begin{tabular}{lrrr}
\hline Settling method & $\Delta f_{\max }[\mathrm{mHz}]$ & $E_{\mathrm{PC}}[\mathrm{GWh}]$ & $E_{\mathrm{SC}}[\mathrm{GWh}]$ \\
\hline Synchronous & 71.8 & 2.27 & 6.26 \\
Asynchronous $(N=5)$ & 15.5 & 0.00 & 1.64 \\
\hline
\end{tabular}

\section{Conclusions}

In the deregulated electrical energy market, network operators have to provide market participants with appropriate incentives to guarantee stable operation of the transmission network. In this paper, it was shown that the currently employed incentive system does not necessarily induce power exchange profiles that contribute to grid stability, due to the fundamental inconsistency between energy-based trade and power-related security objectives. State-of-the-art solutions for tackling this issue can affect market freedom or significantly increase complexity of trade. Therefore, an alternative scheduling concept was proposed that relies on standard market arrangements, but settles energy transactions in an asynchronous way. Openand closed-loop simulations were provided to illustrate that by adopting this method, grid operation can become more robust and the strain on balancing reserves can be reduced considerably.

\section{ACKNOWLEDGEMENTS}

This research is part of the EOS-Regelduurzaam and E-price projects that are funded by SenterNovem/Agentschap NL and the European Community Framework program 7, respectively.

\section{REFERENCES}

[1] P. P. J. van den Bosch, A. Jokic, J. Frunt, W. L. Kling, F. Nobel, P. Boonekamp, W. de Boer, R. M. Hermans, and A. Virag, "Pricebased control of ancillary services for power balancing," European Transactions on Electrical Power, 2010, in press.

[2] UCTE, "Frequency control investigation," Tech. Rep., Aug. 2008. [Online]. Available: www.entsoe.eu/resources/publications/

[3] T. Weißbach and E. Welfonder, "High frequency deviations within the European power system: Origins and proposals for improvement," in Power Systems Conf. Exp., Seattle, WA, USA, March 2009, pp. 1-6.

[4] Tractebel Engineering, "Study of the interactions and dependencies of balancing markets, intraday trade and automatically activated reserves," Tech. Rep., Feb. 2009. [Online]. Available: ec.europa.eu/energy/gas_electricity/studies/

[5] UCTE, "System adequacy forecast 2009-2020," UCTE, Tech. Rep., Jan. 2009. [Online]. Available: www.entsoe.eu/resources/publications/

[6] J. Frunt, I. Lampropoulos, and W. L. Kling, "The impact of electricity market design on periodic network frequency excursions," in Proc. European Energy Market Conf. 2011, Zagreb, Croatia, 2011, submitted.

[7] TenneT, "Operational data," 2009. [Online]. Available: www.tennet.org/english/operational_management/

[8] J.-M. Glachant and F. Lévêque, Electricity Reform in Europe - Towards a Single Energy Market. Cheltenham, GB: Edward Elgar Publishing Ltd, 2009.

[9] P. Sommen and K. Janse, "On the relationship between uniform and recurrent nonuniform discrete-time sampling schemes," IEEE Transactions on Signal Processing, vol. 56, no. 10, pp. 5147-5156, Oct. 2008.

[10] P. Anderson, Power System Control and Stability. New York: IEEE Press, 2003.

[11] ENTSO-E, Load-Frequency Control and Performance, 3rd ed., ENTSO-E, March 2009. [Online]. Available: www.entsoe.eu/resources/publications/

[12] M. Heath, Scientific Computing. New York: McGraw-Hill, 2002.

\section{APPENDIX A \\ DERIVATION OF (11)}

Consider the second scenario in Sect. III, where all controllable generation is uniformly distributed over BRPs $i \in \mathcal{I}_{\mathrm{BRP}}$, and where BRP 0 is responsible for all noncontrollable prosumption (i.e., $\mu_{P, i}(t) \neq 0$ only for $i=0$ ). Let

$$
\bar{P}[k]:=\widetilde{P}[n], \quad \text { for } n \leq \frac{k}{N}<n+1, k \in \mathbb{N},
$$


be the result of up-sampling $\widetilde{P}[n]:=\sum_{i} \widetilde{P}_{i}[n]$ with the strictly positive, integer factor $N$. Using (8)-(9), (10) is rewritten as

$$
\hat{P}[k]=\sum_{i=0}^{N-1} \frac{1+2 i}{2 N^{2}}(\bar{P}[k-N+i]+\bar{P}[k+N-i-1]) .
$$

Thus, $\hat{P}[k]$ is a weighted sum of successive samples of $\bar{P}[k]$, or, equivalently, the result of convolving $\bar{P}[k]$ with a symmetric finite impulse response $h_{k}, k=0, \ldots, 2 N-1$. This impulse response is specified by the vector

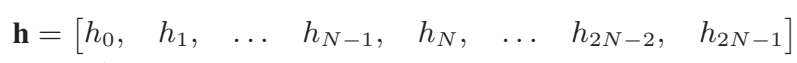

$$
\begin{aligned}
& =\frac{1}{2 N^{2}}[1,1+2, \ldots 1+2(N-1), 1+2(N-1), \ldots 1+2,1] .
\end{aligned}
$$

Given (17), we observe the following. Firstly, note that the sum of the coefficients of $\mathbf{h}$ equals

$$
\sum_{k=0}^{2 N-1} h_{k}=2 \cdot \frac{1}{2 N^{2}}\left(\frac{1+(1+2(N-1))}{2} \cdot N\right)=1 .
$$

This implies that all E-program transactions are contained in the E-references. Secondly, observe that $\mathbf{h}$ describes a $2 N$ wide low-pass digital filter applied to the aggregated controlled power profile for the power system as a whole, which is sampled at a rate of $\frac{N}{T_{\mathrm{PTV}}}$. Hence, under APM scheduling, transaction $E_{i}[n]$ is distributed over 2 PTUs of the market time frame. Both observations are in accordance with Prop. III.3.

\section{APPENDIX B \\ DERIVATION OF PROPOSITION III.4}

\section{A. Synchronized settlement}

An expression for the open-loop power exchange error associated with the currently-employed, $T_{\mathrm{PTU}}$-based synchronous scheduling arrangements, and an upper bound thereon, is derived as follows. Let the aggregated step-wise power prosumption profile be described by

$$
\widetilde{P}[n]:=\frac{1}{T_{\mathrm{PTU}}} \int_{t_{n}}^{t_{n+1}} \mu_{P}(\tau) \mathrm{d} \tau=\frac{E\left(t_{n+1}\right)-E\left(t_{n}\right)}{T_{\mathrm{PTU}}},
$$

where

$$
E(\tau)=\int_{0}^{\tau} \mu_{P}(t) d t+E(0) \quad \Leftrightarrow \quad E^{\prime}(\tau)=\mu_{P}(\tau) .
$$

Assuming that $E(\tau)$ is infinitely differentiable for all $\tau \in \mathbb{R}$, its Taylor series representation around $t \in\left[t_{n}, t_{n+1}\right)$ is

$$
E(\tau)=E(t)+E^{\prime}(t)(\tau-t)+\frac{E^{\prime \prime}(t)}{2}(\tau-t)^{2}+\mathcal{O}\left((\tau-t)^{3}\right),
$$

see, e.g., [12]. From this, it follows that

$$
\begin{aligned}
E\left(t_{n}\right)=E(t)+ & E^{\prime}(t)\left(t_{n}-t\right)+\frac{E^{\prime \prime}(t)}{2}\left(t_{n}-t\right)^{2}+\mathcal{O}\left(\left(t_{n}-t\right)^{3}\right) \\
E\left(t_{n+1}\right)=E(t) & +E^{\prime}(t)\left(t_{n+1}-t\right) \\
& +\frac{E^{\prime \prime}(t)}{2}\left(t_{n+1}-t\right)^{2}+\mathcal{O}\left(\left(t_{n+1}-t\right)^{3}\right) .
\end{aligned}
$$

By combining $t_{n}:=n T_{\mathrm{PTU}}, t_{n+1}:=(n+1) T_{\mathrm{PTU}},(20)$ and the above expressions for $E_{n}$ and $E_{n+1}$, (19) is rewritten as

$$
\begin{aligned}
\widetilde{P}[n]= & \mu_{P}(t)-\frac{1}{2}\left((2 n+1) T_{\mathrm{PTU}}-2 t\right) \mu_{P}^{\prime}(t) \\
& +\frac{1}{6}\left(\left(1+3 n+3 n^{2}\right) T_{\mathrm{PTU}}^{2}-3 t(2 n+1) T_{\mathrm{PTU}}+3 t^{2}\right) \mu_{P}^{\prime \prime}(t) \\
& +\mathcal{O}\left(T_{\mathrm{PTU}}^{3}\right) .
\end{aligned}
$$

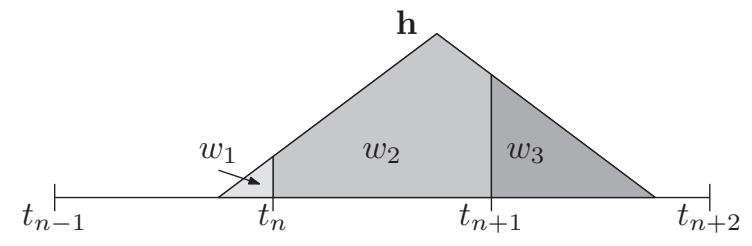

Fig. 9. Impulse response of APM filter $\mathbf{h}$ for $N \rightarrow \infty\left(\frac{T_{\mathrm{PTU}}}{N} \rightarrow 0\right)$.

Introducing $t^{*}:=t-t_{n} \in\left[0, T_{\mathrm{PTU}}\right)$ yields

$$
\begin{aligned}
\widetilde{P}[n]= & \mu_{P}(t)-\frac{1}{2}\left(T_{\mathrm{PTU}}-2 t^{*}\right) \mu_{P}^{\prime}(t) \\
& +\frac{1}{6}\left(T_{\mathrm{PTU}}^{2}-3 t^{*} T_{\mathrm{PTU}}+3\left(t^{*}\right)^{2}\right) \mu_{P}^{\prime \prime}(t)+\mathcal{O}\left(T_{\mathrm{PTU}}^{3}\right) .
\end{aligned}
$$

Hence, it follows that the open-loop error $\Delta P_{\mathrm{OL}}(t):=\widetilde{P}[n]-$ $\mu_{P}(t)$ for $t \in\left[t_{n}, t_{n+1}\right)$ is given by

$$
\begin{aligned}
\Delta P_{\mathrm{OL}}(t) & =-\frac{1}{2}\left(T_{\mathrm{PTU}}-2 t^{*}\right) \mu_{P}^{\prime}(t) \\
& +\frac{1}{6}\left(T_{\mathrm{PTU}}^{2}-3 t^{*} T_{\mathrm{PTU}}+3\left(t^{*}\right)^{2}\right) \mu_{P}^{\prime \prime}(t)+\mathcal{O}\left(T_{\mathrm{PTU}}^{3}\right) .
\end{aligned}
$$

Now, the upper bound on $\left|\Delta P_{\mathrm{OL}}(t)\right|$, i.e., (12), follows by recalling the triangle and Cauchy-Schwarz inequalities, and the fact that $t^{*}:=t-t_{n} \in\left[0, T_{\mathrm{PTU}}\right)$ :

$$
\begin{aligned}
\left|\Delta P_{\mathrm{OL}}(t)\right| \leq & \frac{1}{2}\left|T_{\mathrm{PTU}}-2 t^{*}\right|\left|\mu_{P}^{\prime}(t)\right| \\
& +\frac{1}{6}\left|T_{\mathrm{PTU}}^{2}-3 t^{*} T_{\mathrm{PTU}}+3\left(t^{*}\right)^{2}\right|\left|\mu_{P}^{\prime \prime}(t)\right|+\mathcal{O}\left(T_{\mathrm{PTU}}^{3}\right) \\
\leq & \frac{T_{\mathrm{PTU}}}{2}\left|\mu_{P}^{\prime}(t)\right|+\frac{T_{\mathrm{PTU}}^{2}}{6}\left|\mu_{P}^{\prime \prime}(t)\right|+\mathcal{O}\left(T_{\mathrm{PTU}}^{3}\right) .
\end{aligned}
$$

\section{B. Evenly-distributed APM}

For $N \rightarrow \infty$ and evenly distributed controllable generation/load, the expression for $\Delta P_{\mathrm{OL}}(t)$ under APM is derived as follows. In Appendix A, it was shown that $\hat{P}[k]$ is the result of convolving $\bar{P}[k]$ with discrete-time impulse response $h_{k}$. Fig. 9 shows that for $N \rightarrow \infty$ /infinitesimally small sampling period $\frac{T_{\mathrm{PTU}}}{N}$, this impulse response is triangular with width $2 T_{\text {PTU }}$ and height $\frac{1}{T_{\text {PTU }}}$ (such that (18) holds). Accordingly, it is possible to express $\hat{P}(t)$ for $t \in\left[t_{n}, t_{n+1}\right)$ as a weighted sum of $\widetilde{P}[n-1], \widetilde{P}[n]$ and $\widetilde{P}[n+1]$, i.e.,

$$
\hat{P}(t)=w_{1}(t) \widetilde{P}[n-1]+w_{2}(t) \widetilde{P}[n]+w_{3}(t) \widetilde{P}[n+1],
$$

for $t \in\left[t_{n}, t_{n+1}\right)$, with

$$
\begin{aligned}
& w_{1}(t):=\frac{1}{2}\left(\frac{T_{\mathrm{PTU}}-t^{*}}{T_{\mathrm{PTU}}}\right)^{2}, \quad w_{2}(t):=\frac{3}{4}-\left(\frac{1}{2}-\frac{T_{\mathrm{PTU}}-t^{*}}{T_{\mathrm{PTU}}}\right)^{2}, \\
& w_{3}(t):=\frac{1}{2}\left(\frac{t^{*}}{T_{\mathrm{PTU}}}\right)^{2} .
\end{aligned}
$$

Combining (21) and (25) yields

$$
\hat{P}(t)=\mu_{P}(t)+\frac{T_{\mathrm{PTU}}^{2}}{6} \mu_{P}^{\prime \prime}(t)+\mathcal{O}\left(T_{\mathrm{PTU}}^{3}\right),
$$

such that the open-loop error $\Delta P_{\mathrm{OL}}(t)$ is given by

$$
\Delta P_{\mathrm{OL}}(t)=\hat{P}(t)-\mu_{P}(t)=\frac{T_{\mathrm{PTU}}^{2}}{6} \mu_{P}^{\prime \prime}(t)+\mathcal{O}\left(T_{\mathrm{PTU}}^{3}\right) .
$$

Now, the upper bound on $\left|\Delta P_{\mathrm{OL}}(t)\right|$, i.e., (13), follows:

$$
\left|\Delta P_{\mathrm{OL}}(t)\right| \leq \frac{T_{\mathrm{PTU}}^{2}}{6}\left|\mu_{P}^{\prime \prime}(t)\right|+\mathcal{O}\left(T_{\mathrm{PTU}}^{3}\right) .
$$

